RETRACTION

doi:10.1038/nature13549

\title{
Retraction: Generation of cell polarity in plants links endocytosis, auxin distribution and cell fate decisions
}

Pankaj Dhonukshe, Hirokazu Tanaka, Tatsuaki Goh, Kazuo Ebine, Ari Pekka Mähönen, Kalika Prasad, Ikram Blilou, Niko Geldner, Jian Xu, Tomohiro Uemura, Joanne Chory, Takashi Ueda, Akihiko Nakano, Ben Scheres \& Jiří Friml

Nature 456, 962-966 (2008); doi:10.1038/nature07409

Our Letter reported that PIN transporters for the plant hormone auxin are initially delivered to the plasma membrane in a non-polar manner and that their polar distribution requires endocytosis. Abolishing PIN polarization, such as by inhibiting endocytosis, interferes with local auxin responses in the embryo, leading to transformation of embryonic leaves to the root-like structures.

The data regarding the essential role of endocytosis in the PIN polar localization and the connection between PIN polarity, auxin distribution and cell fate decisions remain reliable, but we have come to realize that the interpretation concerning the initial non-polar delivery of PIN proteins to the plasma membrane is not fully supported by experiments. It concerns the fluorescence recovery after photobleaching (FRAP) experiments presented in Fig. 1a and Supplementary Fig. 2a, which provided the key suggestion for the non-polar delivery model. On the basis of the original data, we confirm that the experiments were performed as published, but despite multiple attempts to reproduce them, the results remain inconclusive. Although in some experiments nonpolar recovery can be detected as reported, others yield contrasting outputs that suggest polar recovery. Importantly, in many cases, the cells show signs of severe stress and stop growing following the photobleaching. In light of these findings, we feel that the reported results cannot be used for the conclusion on the initial non-polar PIN delivery and that this question remains open.

Therefore, we prefer to retract this Letter and republish the remaining confirmed findings elsewhere. Author P.D. continues to stand by all the conclusions of the paper, but all the other authors agree with the retraction. We apologise for any adverse consequences that may have resulted from this situation. 